\title{
Photoionization cross sections and oscillator strengths for Fe III
}

\author{
Sultana N. Nahar \\ Department of Astronomy, The Ohio State University, Columbus, Ohio 43210
}

(Received 12 October 1995)

\begin{abstract}
$A b$ initio calculations are carried out for the photoionization cross sections and oscillator strengths for $\mathrm{Fe}$ III in the close-coupling approximation employing the $R$-matrix method. A 49-state eigenfunction expansion for Fe IV, with states dominated by the ground configuration $3 d^{5}$ and the excited configurations $3 d^{4} 4 s$ and $3 d^{4} 4 p$, is employed to ensure an extensive treatment of autoionizing resonances and other electron correlation effects related to channel couplings that considerably affect the effective cross sections. Of particular interest are the wide resonances due to photoexcitation within the core states that enhance the background cross sections by orders of magnitude at high energies. Coupled wave functions are obtained for 805 bound states of Fe III below the first ionization threshold; all of the 199 LS terms observed experimentally have been identified. Photoionization cross sections, with detailed autoionizing resonances, are obtained for the computed bound states as a function of photoelectron energy. The present results also include the oscillator strengths for 11979 transitions between the bound states of Fe III that lie below the ionization threshold. In addition, the partial photoionization cross sections for 239 bound states, with the residual ion in the ground state, are also obtained. These cross sections are computed for applications to the determination of electron-ion recombination rate coefficients and population of levels in collisional-radiative models that do not assume local thermodynamic equilibrium. The present radiative data are compared with the observed energy values, lifetimes, and the photoionization cross sections calculated by others.
\end{abstract}

PACS number(s): 32.80.Fb

\section{INTRODUCTION}

Fe III is a very important ion in astrophysical sources but its detailed theoretical study is difficult because of its complexity. The strong electron correlation effects in this 24electron ion require a large eigenfunction expansion for an accurate wave-function representation. In a previous calculation under the Opacity Project (OP) [1], the radiative data for energy levels, photoionization cross sections, and oscillator strengths were obtained for Fe III [2] employing a relatively limited eigenfunction expansion with the 16 terms dominated by only the ground configuration $3 d^{5}$. As we show in this work, this expansion is not quite adequate to achieve the desired accuracy or completeness of the set of radiative quantities for Fe III. Consequently the OP data set requires substantial improvement in terms of missing states, corresponding oscillator strengths for bound-bound transitions, and absence of important resonances in photoionization cross sections, etc. The aim of the present work is to study Fe III in much greater detail and obtain more accurate radiative data. The present work forms part of the new Iron Project (IP) [3], which, in addition to collisional calculations (which is its primary aim), also involves improvement of some radiative data for complex atomic systems not adequately treated in the OP work. Recent studies of large-scale computations of Fe II [4] and of Fe I [5] show that the photoionization cross sections in the new calculations can differ considerably, even by orders of magnitude, from earlier calculations in either simpler atomic physics approximations, such as central field, Hartree-Slater, single configuration Hartree-Fock, or even limited close-coupling calculations that do not include all contributing channels. On the other hand, for practical applications it is important to obtain these parameters to high accuracy. For example, the abundances and ionization frac- tions of iron in astrophysical sources depend critically on photoionization and recombination parameters that might possibly explain the observed spectra of stars, gaseous nebulae, quasars, etc.

In this paper we present selected results for the energies, photoionization cross sections, and oscillator strengths of Fe III, illustrating the physical effects that manifest themselves in a large coupled-channel calculation of radiative processes. Of particular interest are the two main types of resonances in the cross sections, one due to the Rydberg series of quasibound states, above the ionization threshold, converging onto the excited target thresholds of Fe IV, and the other type due to the photoexcitation of a dipole transition from the ground state of Fe IV, i.e., $3 d^{5}\left({ }^{6} S\right) \rightarrow 3 d^{4} 4 p\left({ }^{6} P^{o}\right)$. The second type of resonance is the photoexcitation-of-core (PEC) resonance and is intimately connected with the inverse dielectronic recombination process $[6,7]$. The PEC resonances are not included in any earlier work (the previous OP calculation did not include the excited $3 d^{5} 4 p$ configuration). The total number of bound states and oscillator strengths obtained are much larger than the previous OP data. The present work also provides the partial photoionization cross sections for ionization into the ground state of the residual ion. The partial cross sections are directly applicable to ongoing work on total, unified election-ion recombination rates [7], and astrophysical diagnostics.

\section{COMPUTATIONS}

In the close-coupling approximation $(\mathrm{CC})$, the core ion, termed the "target," is represented by an $N$-electron system and the wave-function expansion $\Psi(E)$ for any symmetry, $S L \pi$, of the total $(N+1)$-electron system is represented in 
terms of the core or the target ion states as

$$
\Psi(E)=A \sum_{i} \chi_{i} \theta_{i}+\sum_{j} c_{j} \Phi_{j}
$$

where $\chi_{i}$ is the target ion wave function in a specific state $S_{i} L_{i} \pi_{i}$ and $\theta_{i}$ is the wave function for the $(N+1)$ th electron in a channel labeled as $S_{i} L_{i} \pi_{i} k_{i}^{2} \ell_{i}(S L \pi) ; k_{i}^{2}$ is its incident kinetic energy. $\Phi_{j}$ 's are the correlation wave functions of the $(N+1)$-electron system that compensates the orthogonality condition of the total wave function. The target state wave functions for Fe IV is obtained from the atomic structure computer code SUPERSTRUCTURE [8], prior to the $R$-matrix calculations. The 49-state eigenfunction expansion is chosen such that it includes the dominant dipole transitions in the core and important correlation effects. Comparison of the calculated energies of the 49 states with the observed energies is presented in Table I. The calculated energies agree with the observed values usually within a few percent, with a few exceptions; the largest difference is $17.5 \%$ for the state $3 d^{5}\left({ }^{2} I\right)$. This indicates an overall reasonably good agreement of the calculated values with the observed ones in light of the complex atomic structure involved. The table also includes the list of principal and correlation configurations employed for the target ion and the values of the scaling parameters $\lambda_{n \ell}$, for the set of target orbitals $n \ell$, in the scaled Thomas-Fermi-Dirac potential in SUPERSTRUCTURE.

One crucial factor for proper representation of $\Psi(E)$ is the second sum in the wave-function expansion that accounts for orthogonality constraints between the bound target and the free continuum orbitals, as well as for additional shortrange electron correlation. For smaller atomic systems the sum can include all possible $(N+1)$-electron states with the chosen number of the target orbital set $n \ell$. For large atomic systems, such as Fe III, however, such a sum may correspond to a very large number of states that may not all be important but result in a huge Hamiltonian matrix to be diagonalized. Hence a judicious choice needs to be made for the set of $(N+1)$-electron correlation functions that are significant. We employ a different, suitably optimized, set of such functions for each total spin symmetry of the electron-ion system; we consider $(2 S+1)=1,3,5,7$. For a given total spin, only those target states coupled to that spin value are included in the $\mathrm{CC}$ expansion, thus significantly reducing the size of the calculations.

The CC calculations for Fe III are carried out in a manner similar to the OP [1] calculations. The theory is explained in Ref. [1]. The computations are carried out using the $R$-matrix codes developed for the OP [9], but extended for the IP work [3]. The calculations are, essentially, as in the earlier large-scale computations for the radiative data of Fe II [4]. Some modifications were made to the asymptotic code of the $R$-matrix package that computes the bound-free transition amplitudes to yield partial, state-specific (with given initial and final states), photoionization cross sections.

Calculations of Fe III are carried out in $L S$ coupling, with the assumption that the relativistic effects may not be considerable because of the low ion charge. The present work considers all bound states of $S_{t} L_{t} \pi_{t} n \ell$, where $S_{t} L_{t} \pi_{t}$ are the 49 target states and $n \leqslant 10$ and $0 \leqslant \ell \leqslant n-1$. It is nontrivial to carry out the spectroscopic identification of the calculated
TABLE I. The Fe IV target states in the 49-state CC expansion for Fe III. $E$ (obs) corresponds to the observed [12] and $E$ (calc) to the calculated energies in Ry. Principal configurations: $3 s^{2} 3 p^{6} 3 d^{5}, 3 s^{2} 3 p^{6} 3 d^{4} 4 s$, and $3 s^{2} 3 p^{6} 3 d^{4} 4 p$. Correlation configurations: $\quad 3 s^{2} 3 p^{5} 3 d^{6}, \quad 3 s^{2} 3 p^{4} 3 d^{7}, \quad 3 s^{2} 3 p^{6} 3 d^{3} 4 s 4 p$, $2 p^{6} 3 p^{6} 3 d^{7}, 3 s 3 p^{6} 3 d^{5} 4 s, 3 s^{2} 3 p^{6} 3 d^{3} 4 s 4 d$, and $3 s 3 p^{6} 3 d^{4} 4 s^{2}$. Thomas-Fermi scaling parameters: $1.40467(1 s), 1.11279(2 s)$, $1.27320(2 p), 1.27320(3 s), 1.07320(3 p), 1.05832(3 d), 1.07320$ $(4 s), 1.05832(4 p)$, and 1.40794(4d).

\begin{tabular}{|c|c|c|c|}
\hline Configuration & Term & $E(\mathrm{obs})$ & $E($ calc $)$ \\
\hline $3 d^{5}$ & ${ }^{6} S^{e}$ & 0.000000 & 0.000000 \\
\hline $3 d^{5}$ & ${ }^{4} G^{e}$ & 0.294169 & 0.343447 \\
\hline $3 d^{5}$ & ${ }^{4} P^{e}$ & 0.321730 & 0.349341 \\
\hline $3 d^{5}$ & ${ }^{4} D^{e}$ & 0.354206 & 0.406702 \\
\hline $3 d^{5}$ & ${ }^{2} I^{e}$ & 0.429070 & 0.504217 \\
\hline $3 d^{5}$ & ${ }^{2} D^{e}$ & 0.453314 & 0.504982 \\
\hline $3 d^{5}$ & ${ }^{2} F^{e}$ & 0.471355 & 0.517330 \\
\hline $3 d^{5}$ & ${ }^{4} F^{e}$ & 0.480416 & 0.538692 \\
\hline $3 d^{5}$ & ${ }^{2} H^{e}$ & 0.512384 & 0.576651 \\
\hline $3 d^{5}$ & ${ }^{2} G^{e}$ & 0.524726 & 0.603606 \\
\hline $3 d^{5}$ & ${ }^{2} F^{e}$ & 0.557809 & 0.639729 \\
\hline $3 d^{5}$ & ${ }^{2} S^{e}$ & 0.607998 & 0.694126 \\
\hline $3 d^{5}$ & ${ }^{2} D^{e}$ & 0.675418 & 0.755665 \\
\hline $3 d^{5}$ & ${ }^{2} G^{e}$ & 0.755404 & 0.856408 \\
\hline $3 d^{5}$ & ${ }^{2} P^{e}$ & 0.912367 & 1.031666 \\
\hline $3 d^{5}$ & ${ }^{2} D^{e}$ & 0.986434 & 1.095135 \\
\hline $3 d^{4}\left({ }^{5} D\right) 4 s$ & ${ }^{6} D^{e}$ & 1.170799 & 1.159025 \\
\hline $3 d^{4}\left({ }^{5} D\right) 4 s$ & ${ }^{4} D^{e}$ & 1.261186 & 1.264731 \\
\hline $3 d^{4}\left({ }^{3} H\right) 4 s$ & ${ }^{4} H^{e}$ & 1.407729 & 1.420342 \\
\hline $3 d^{4}\left({ }^{3} P_{2}\right) 4 s$ & ${ }^{4} P^{e}$ & 1.412215 & 1.439247 \\
\hline $3 d^{4}\left({ }^{3} F_{2}\right) 4 s$ & ${ }^{4} F^{e}$ & 1.422743 & 1.445444 \\
\hline $3 d^{4}\left({ }^{3} G\right) 4 s$ & ${ }^{4} G^{e}$ & 1.450157 & 1.470624 \\
\hline $3 d^{4}\left({ }^{3} H\right) 4 s$ & ${ }^{2} H^{e}$ & 1.463188 & 1.482829 \\
\hline $3 d^{4}\left({ }^{3} P_{2}\right) 4 s$ & ${ }^{2} P^{e}$ & 1.467623 & 1.501715 \\
\hline $3 d^{4}\left({ }^{3} F_{2}\right) 4 s$ & ${ }^{2} F^{e}$ & 1.477001 & 1.508756 \\
\hline $3 d^{4}\left({ }^{3} G\right) 4 s$ & ${ }^{2} G^{e}$ & 1.505378 & 1.533146 \\
\hline $3 d^{4}\left({ }^{3} D\right) 4 s$ & ${ }^{4} D^{e}$ & 1.509078 & 1.541497 \\
\hline $3 d^{4}\left({ }^{1} G_{2}\right) 4 s$ & ${ }^{2} G^{e}$ & 1.528647 & 1.558940 \\
\hline $3 d^{4}\left({ }^{1} I\right) 4 s$ & ${ }^{2} I^{e}$ & 1.535894 & 1.559382 \\
\hline $3 d^{4}\left({ }^{1} S_{2}\right) 4 s$ & ${ }^{2} S^{e}$ & 1.555802 & 1.598304 \\
\hline $3 d^{4}\left({ }^{3} D\right) 4 s$ & ${ }^{2} D^{e}$ & 1.561891 & 1.605603 \\
\hline $3 d^{4}\left({ }^{1} D_{2}\right) 4 s$ & ${ }^{2} D^{e}$ & 1.613219 & 1.664325 \\
\hline $3 d^{4}\left({ }^{1} F\right) 4 s$ & ${ }^{2} F^{e}$ & 1.669099 & 1.723453 \\
\hline $3 d^{4}\left({ }^{5} D\right) 4 p$ & ${ }^{6} F^{o}$ & 1.724550 & 1.818157 \\
\hline $3 d^{4}\left({ }^{5} D\right) 4 p$ & ${ }^{6} P^{o}$ & 1.732119 & 1.831152 \\
\hline $3 d^{4}\left({ }^{3} F_{1}\right) 4 s$ & ${ }^{4} F^{e}$ & 1.734927 & 1.807848 \\
\hline $3 d^{4}\left({ }^{3} P_{1}\right) 4 s$ & ${ }^{4} P^{e}$ & 1.735791 & 1.810057 \\
\hline $3 d^{4}\left({ }^{5} D\right) 4 p$ & ${ }^{4} P^{o}$ & 1.754276 & 1.862131 \\
\hline $3 d^{4}\left({ }^{5} D\right) 4 p$ & ${ }^{6} D^{o}$ & 1.761746 & 1.861259 \\
\hline $3 d^{4}\left({ }^{3} F_{1}\right) 4 s$ & ${ }^{2} F^{e}$ & 1.787629 & 1.871164 \\
\hline $3 d^{4}\left({ }^{3} P_{1}\right) 4 s$ & ${ }^{2} P^{e}$ & 1.787918 & 1.874124 \\
\hline $3 d^{4}\left({ }^{5} D\right) 4 p$ & ${ }^{4} F^{o}$ & 1.791167 & 1.904812 \\
\hline $3 d^{4}\left({ }^{1} G_{1}\right) 4 s$ & ${ }^{2} G^{e}$ & 1.833408 & 1.915998 \\
\hline $3 d^{4}\left({ }^{5} D\right) 4 p$ & ${ }^{4} D^{o}$ & 1.843957 & 1.968479 \\
\hline $3 d^{4}\left({ }^{3} H\right) 4 p$ & ${ }^{4} H^{o}$ & 1.938032 & 2.040010 \\
\hline $3 d^{4}\left({ }^{3} P_{2}\right) 4 p$ & ${ }^{4} D^{o}$ & 1.953335 & 2.073489 \\
\hline $3 d^{4}\left({ }^{3} F_{2}\right) 4 p$ & ${ }^{4} G^{o}$ & 1.963043 & 2.077942 \\
\hline $3 d^{4}\left({ }^{3} H\right) 4 p$ & ${ }^{4} I^{o}$ & 1.969819 & 2.070261 \\
\hline $3 d^{4}\left({ }^{3} H\right) 4 p$ & ${ }^{2} G^{o}$ & 1.970958 & 2.081440 \\
\hline
\end{tabular}


TABLE II. Comparison of the calculated energies, $E$ (calc), of Fe III with the observed ones $E$ (obs) [12,13]. "Ek" indicates energy measured by Ekberg [13]. All energies are in Rydberg units. The ionization potential is 2.2528 Ry.

\begin{tabular}{|c|c|c|c|c|c|c|c|}
\hline Configuration & $S L \pi$ & $E(\mathrm{obs})$ & $E($ calc $)$ & Configuration & $S L \pi$ & $E(\mathrm{obs})$ & $E($ calc $)$ \\
\hline $3 d^{5}\left({ }^{6} S\right) 4 s$ & ${ }^{7} S^{e}$ & 1.97864 & 1.928 & $3 d^{5}\left({ }^{6} S\right) 4 p$ & ${ }^{7} P^{o}$ & 1.50127 & 1.485 \\
\hline $3 d^{5}\left({ }^{6} S\right) 4 d$ & ${ }^{7} D^{e}$ & 0.91033 & 0.893 & $3 d^{5}\left({ }^{6} S\right) 5 s$ & ${ }^{7} S^{e}$ & 0.89244 & 0.884 \\
\hline $3 d^{5}\left({ }^{6} S\right) 5 p$ & ${ }^{7} P^{o}$ & 0.73740 & 0.726 & $3 d^{5}\left({ }^{6} S\right) 4 f$ & ${ }^{7} F^{o \mathrm{a}}$ & 0.57272 & 0.575 \\
\hline $3 d^{4}\left({ }^{5} D\right) 4 s 4 p^{3} P^{o}$ & ${ }^{7} P^{o}$ & 0.54930 & 0.560 & $3 d^{5}\left({ }^{6} S\right) 5 d$ & ${ }^{7} D^{e}$ & 0.51767 & 0.511 \\
\hline $3 d^{5}\left({ }^{6} S\right) 6 s$ & ${ }^{7} S^{e}$ & 0.51306 & 0.510 & $3 d^{5}\left({ }^{6} S\right) 6 p$ & ${ }^{7} P^{o}$ & 0.44233 & 0.437 \\
\hline $3 d^{5}\left({ }^{6} S\right) 5 f$ & ${ }^{7} F^{o \mathrm{a}}$ & 0.36542 & 0.364 & $3 d^{5}\left({ }^{6} S\right) 5 g$ & ${ }^{7} G^{e \mathrm{a}}$ & 0.36067 & 0.360 \\
\hline $3 d^{5}\left({ }^{6} S\right) 6 d$ & ${ }^{7} D^{e}$ & 0.33549 & 0.332 & $3 d^{5}\left({ }^{6} S\right) 7 s$ & ${ }^{7} S^{e}$ & 0.33356 & 0.332 \\
\hline $3 d^{5}\left({ }^{6} S\right) 6 g$ & ${ }^{7} G^{e \mathrm{a}}$ & 0.25041 & 0.250 & $3 d^{5}\left({ }^{6} S\right) 6 h$ & ${ }^{7} H^{o \mathrm{a}}$ & 0.25005 & 0.250 \\
\hline $3 d^{6}$ & ${ }^{5} D^{e}$ & 2.24898 & 2.127 & $3 d^{5}\left({ }^{6} S\right) 4 s$ & ${ }^{5} S^{e}$ & 1.87921 & 1.828 \\
\hline $3 d^{5}\left({ }^{4} G\right) 4 s$ & ${ }^{5} G^{e}$ & 1.67448 & 1.586 & $3 d^{5}\left({ }^{4} P\right) 4 s$ & ${ }^{5} P^{e}$ & 1.64675 & 1.582 \\
\hline $3 d^{5}\left({ }^{4} D\right) 4 s$ & ${ }^{5} D^{e}$ & 1.61698 & 1.532 & $3 d^{5}\left({ }^{4} F\right) 4 s$ & ${ }^{5} F^{e}$ & 1.49430 & 1.407 \\
\hline $3 d^{5}\left({ }^{6} S\right) 4 p$ & ${ }^{5} P^{o}$ & 1.43953 & 1.421 & $3 d^{5}\left({ }^{4} G\right) 4 p$ & ${ }^{5} G^{o}$ & 1.21704 & 1.148 \\
\hline $3 d^{5}\left({ }^{4} G\right) 4 p$ & ${ }^{5} H^{o}$ & 1.20162 & 1.137 & $3 d^{5}\left({ }^{4} G\right) 4 p$ & ${ }^{5} F^{o}$ & 1.19089 & 1.122 \\
\hline $3 d^{5}\left({ }^{4} P\right) 4 p$ & ${ }^{5} S^{o}$ & 1.18758 & 1.137 & $3 d^{5}\left({ }^{4} P\right) 4 p$ & ${ }^{5} D^{o}$ & 1.18673 & 1.136 \\
\hline $3 d^{5}\left({ }^{4} P\right) 4 p$ & ${ }^{5} P^{o}$ & 1.17188 & 1.119 & $3 d^{5}\left({ }^{4} D\right) 4 p$ & ${ }^{5} F^{o}$ & 1.14874 & 1.075 \\
\hline $3 d^{5}\left({ }^{4} D\right) 4 p$ & ${ }^{5} D^{o}$ & $1.13378 \mathrm{Ek}$ & 1.060 & $3 d^{5}\left({ }^{4} D\right) 4 p$ & ${ }^{5} P^{o}$ & 1.12566 & 1.056 \\
\hline $3 d^{5}\left({ }^{4} F\right) 4 p$ & ${ }^{5} G^{o}$ & 1.01894 & 0.940 & $3 d^{5}\left({ }^{4} F\right) 4 p$ & ${ }^{5} F^{o}$ & 1.01264 & 0.930 \\
\hline $3 d^{5}\left({ }^{4} F\right) 4 p$ & ${ }^{5} D^{o}$ & 1.00081 & 0.919 & $3 d^{5}\left({ }^{6} S\right) 4 d$ & ${ }^{5} D^{e}$ & 0.87193 & 0.853 \\
\hline $3 d^{5}\left({ }^{6} S\right) 5 s$ & ${ }^{5} S^{e}$ & 0.86991 & 0.860 & $3 d^{5}\left({ }^{6} S\right) 5 p$ & ${ }^{5} P^{o}$ & 0.71835 & 0.708 \\
\hline $3 d^{5}\left({ }^{4} G\right) 4 d$ & ${ }^{5} H^{e}$ & 0.61977 & 0.574 & $3 d^{5}\left({ }^{4} G\right) 4 d$ & ${ }^{5} F^{e}$ & 0.61590 & 0.571 \\
\hline $3 d^{5}\left({ }^{4} G\right) 4 d$ & ${ }^{5} G^{e}$ & 0.61486 & 0.569 & $3 d^{5}\left({ }^{4} G\right) 4 d$ & ${ }^{5} I^{e}$ & 0.61351 & 0.570 \\
\hline $3 d^{5}\left({ }^{4} G\right) 5 s$ & ${ }^{5} G^{e}$ & 0.59608 & 0.586 & $3 d^{5}\left({ }^{4} P\right) 4 d$ & ${ }^{5} F^{e}$ & 0.59045 & 0.555 \\
\hline $3 d^{5}\left({ }^{6} S\right) 4 f$ & ${ }^{5} F^{o}$ & 0.56900 & 0.565 & $3 d^{5}\left({ }^{4} P\right) 5 s$ & ${ }^{5} P^{e}$ & 0.56707 & 0.559 \\
\hline $3 d^{5}\left({ }^{4} D\right) 4 d$ & ${ }^{5} G^{e}$ & 0.55390 & 0.510 & $3 d^{5}\left({ }^{4} D\right) 4 d$ & ${ }^{5} D^{e \mathrm{a}}$ & 0.54980 & 0.506 \\
\hline $3 d^{5}\left({ }^{4} D\right) 5 s$ & ${ }^{5} D^{e}$ & 0.53888 & 0.526 & $3 d^{5}\left({ }^{6} S\right) 6 s$ & ${ }^{5} S^{e}$ & 0.50313 & 0.498 \\
\hline $3 d^{5}\left({ }^{6} S\right) 5 d$ & ${ }^{5} D^{e}$ & 0.48854 & 0.462 & $3 d^{5}\left({ }^{4} G\right) 5 p$ & ${ }^{5} G^{o}$ & 0.44546 & 0.431 \\
\hline $3 d^{5}\left({ }^{4} G\right) 5 p$ & ${ }^{5} H^{o}$ & 0.44140 & 0.428 & $3 d^{5}\left({ }^{4} G\right) 5 p$ & ${ }^{5} F^{o}$ & 0.43738 & 0.424 \\
\hline $3 d^{5}\left({ }^{4} F\right) 4 d$ & ${ }^{5} H^{e}$ & 0.43134 & 0.379 & $3 d^{5}\left({ }^{4} F\right) 4 d$ & ${ }^{5} G^{e}$ & 0.42611 & 0.378 \\
\hline $3 d^{5}\left({ }^{4} P\right) 5 p$ & ${ }^{5} D^{o}$ & 0.41952 & 0.402 & $3 d^{5}\left({ }^{4} P\right) 5 p$ & ${ }^{5} S^{o}$ & 0.41851 & 0.404 \\
\hline $3 d^{5}\left({ }^{4} F\right) 5 s$ & ${ }^{5} F^{e}$ & 0.41197 & 0.400 & $3 d^{5}\left({ }^{4} P\right) 5 p$ & ${ }^{5} P^{o}$ & 0.40975 & 0.399 \\
\hline $3 d^{5}\left({ }^{4} D\right) 5 p$ & ${ }^{5} F^{o}$ & 0.38410 & 0.369 & $3 d^{5}\left({ }^{4} D\right) 5 p$ & ${ }^{5} D^{o \mathrm{a}}$ & 0.37830 & 0.366 \\
\hline $3 d^{5}\left({ }^{6} S\right) 5 f$ & ${ }^{5} F^{o}$ & 0.36408 & 0.362 & $3 d^{5}\left({ }^{6} S\right) 5 g$ & ${ }^{5} G^{e}$ & 0.36065 & 0.360 \\
\hline $3 d^{5}\left({ }^{4} F\right) 5 p$ & ${ }^{5} G^{o}$ & 0.25676 & 0.243 & $3 d^{5}\left({ }^{4} F\right) 5 p$ & ${ }^{5} F^{o}$ & 0.25239 & 0.243 \\
\hline $3 d^{5}\left({ }^{6} S\right) 6 g$ & ${ }^{5} G^{e}$ & 0.25039 & 0.250 & $3 d^{5}\left({ }^{6} S\right) 6 h$ & ${ }^{5} H^{o \mathrm{a}}$ & 0.25004 & 0.250 \\
\hline $3 d^{5}\left({ }^{4} G\right) 5 d$ & ${ }^{5} H^{e}$ & 0.22433 & 0.210 & $3 d^{5}\left({ }^{4} G\right) 5 d$ & ${ }^{5} F^{e}$ & 0.22311 & 0.209 \\
\hline $3 d^{5}\left({ }^{4} G\right) 5 d$ & ${ }^{5} G^{e}$ & 0.22303 & 0.209 & $3 d^{5}\left({ }^{4} G\right) 5 d$ & ${ }^{5} I^{e}$ & 0.22234 & 0.209 \\
\hline $3 d^{5}\left({ }^{4} G\right) 6 s$ & ${ }^{5} G^{e}$ & 0.21790 & 0.214 & $3 d^{5}\left({ }^{4} P\right) 6 s$ & ${ }^{5} P^{e}$ & 0.18946 & 0.187 \\
\hline $3 d^{5}\left({ }^{4} D\right) 6 s$ & ${ }^{5} D^{e \mathrm{a}}$ & 0.16155 & 0.154 & $3 d^{6}$ & ${ }^{3} P^{e} 2$ & 2.07027 & 1.952 \\
\hline $3 d^{6}$ & ${ }^{3} H^{e}$ & 2.06828 & 1.920 & $3 d^{6}$ & ${ }^{3} F^{e} 2$ & 2.05567 & 1.926 \\
\hline $3 d^{6}$ & ${ }^{3} G^{e}$ & 2.02649 & 1.885 & $3 d^{6}$ & ${ }^{3} D^{e}$ & 1.97230 & 1.840 \\
\hline $3 d^{6}$ & ${ }^{3} P^{e} 1$ & 1.79726 & 1.665 & $3 d^{6}$ & ${ }^{3} F^{e} 1$ & 1.79482 & 1.656 \\
\hline $3 d^{5}\left({ }^{4} G\right) 4 s$ & ${ }^{3} G^{e}$ & 1.60844 & 1.511 & $3 d^{5}\left({ }^{4} P\right) 4 s$ & ${ }^{3} P^{e}$ & 1.58039 & 1.508 \\
\hline $3 d^{5}\left({ }^{4} D\right) 4 s$ & ${ }^{3} D^{e}$ & 1.55089 & 1.460 & $3 d^{5}\left({ }^{2} I\right) 4 s$ & ${ }^{3} I^{e}$ & 1.52521 & 1.405 \\
\hline $3 d^{5}\left({ }^{2} D_{3}\right) 4 s$ & ${ }^{3} D^{e}$ & 1.50181 & 1.416 & $3 d^{5}\left({ }^{2} F_{2}\right) 4 s$ & ${ }^{3} F^{e}$ & 1.48390 & 1.400 \\
\hline $3 d^{5}\left({ }^{2} H\right) 4 s$ & ${ }^{3} H^{e}$ & 1.44384 & 1.339 & $3 d^{5}\left({ }^{2} G_{2}\right) 4 s$ & ${ }^{3} G^{e}$ & 1.43440 & 1.322 \\
\hline $3 d^{5}\left({ }^{4} F\right) 4 s$ & ${ }^{3} F^{e}$ & 1.42845 & 1.334 & $3 d^{5}\left({ }^{2} F_{1}\right) 4 s$ & ${ }^{3} F^{e}$ & 1.40174 & 1.297 \\
\hline $3 d^{5}\left({ }^{2} S\right) 4 s$ & ${ }^{3} S^{e}$ & 1.35375 & 1.250 & $3 d^{5}\left({ }^{2} D_{2}\right) 4 s$ & ${ }^{3} D^{e}$ & 1.28766 & 1.186 \\
\hline $3 d^{5}\left({ }^{2} G_{1}\right) 4 s$ & ${ }^{3} G^{e}$ & 1.21091 & 1.090 & $3 d^{5}\left({ }^{4} G\right) 4 p$ & ${ }^{3} F^{o}$ & 1.17506 & 1.127 \\
\hline $3 d^{5}\left({ }^{4} G\right) 4 p$ & ${ }^{3} H^{o}$ & 1.17286 & 1.127 & $3 d^{5}\left({ }^{4} P\right) 4 p$ & ${ }^{3} P^{o}$ & 1.16071 & 1.132 \\
\hline $3 d^{5}\left({ }^{4} G\right) 4 p$ & ${ }^{3} G^{o}$ & 1.14164 & 1.090 & $3 d^{5}\left({ }^{4} P\right) 4 p$ & ${ }^{3} D^{o}$ & 1.13616 & 1.108 \\
\hline $3 d^{5}\left({ }^{4} D\right) 4 p$ & ${ }^{3} D^{o}$ & 1.11474 & 1.070 & $3 d^{5}\left({ }^{4} D\right) 4 p$ & ${ }^{3} F^{o}$ & 1.10862 & 1.061 \\
\hline $3 d^{5}\left({ }^{4} P\right) 4 p$ & ${ }^{3} S^{o}$ & 1.10107 & 1.069 & $3 d^{5}\left({ }^{4} D\right) 4 p$ & ${ }^{3} P^{o}$ & 1.07955 & 1.028 \\
\hline
\end{tabular}


TABLE II. (Continued).

\begin{tabular}{|c|c|c|c|c|c|c|c|}
\hline Configuration & $S L \pi$ & $E(\mathrm{obs})$ & $E($ calc $)$ & Configuration & $S L \pi$ & $E(\mathrm{obs})$ & $E($ calc $)$ \\
\hline $3 d^{5}\left({ }^{2} I\right) 4 p$ & ${ }^{3} K^{o}$ & 1.06551 & 0.998 & $3 d^{5}\left({ }^{2} I\right) 4 p$ & ${ }^{3} I^{o}$ & 1.06160 & 0.993 \\
\hline $3 d^{5}\left({ }^{2} P\right) 4 s$ & ${ }^{3} P^{e}$ & $1.06035 \mathrm{Ek}$ & 0.943 & $3 d^{5}\left({ }^{2} D\right) 4 p$ & ${ }^{3} F^{o}$ & 1.04642 & 1.007 \\
\hline $3 d^{5}\left({ }^{2} I\right) 4 p$ & ${ }^{3} H^{o}$ & 1.04566 & 0.973 & $3 d^{5}\left(a^{2} D\right) 4 p$ & ${ }^{3} P^{o}$ & 1.02762 & 0.981 \\
\hline $3 d^{5}\left(a^{2} F\right) 4 p$ & ${ }^{3} G^{o}$ & 1.02083 & 0.978 & $3 d^{5}\left(a^{2} D\right) 4 p$ & ${ }^{3} D^{o}$ & 1.02148 & 0.974 \\
\hline $3 d^{5}\left(a^{2} F\right) 4 p$ & ${ }^{3} D^{o}$ & 1.01150 & 0.963 & $3 d^{5}\left(a^{2} F\right) 4 p$ & ${ }^{3} F^{o}$ & 1.00754 & 0.967 \\
\hline $3 d^{5}\left({ }^{2} H\right) 4 p$ & ${ }^{3} H^{o}$ & 0.99622 & 0.938 & $3 d^{5}\left({ }^{2} H\right) 4 p$ & ${ }^{3} G^{o}$ & 0.99432 & 0.945 \\
\hline $3 d^{5}\left(c^{2} D\right) 4 s$ & ${ }^{3} D^{e}$ & $0.98731 \mathrm{Ek}$ & 0.872 & $3 d^{5}\left({ }^{4} F\right) 4 p$ & ${ }^{3} G^{o}$ & 0.98094 & 0.923 \\
\hline $3 d^{5}\left({ }^{2} H\right) 4 p$ & ${ }^{3} I^{o}$ & 0.97810 & 0.927 & $3 d^{5}\left(a^{2} G\right) 4 p$ & ${ }^{3} F^{o}$ & 0.96940 & 0.913 \\
\hline $3 d^{5}\left({ }^{4} F\right) 4 p$ & ${ }^{3} D^{o}$ & 0.96389 & 0.912 & $3 d^{5}\left({ }^{4} F\right) 4 p$ & ${ }^{3} F^{o}$ & 0.95654 & 0.892 \\
\hline $3 d^{5}\left(a^{2} G\right) 4 p$ & ${ }^{3} H^{o}$ & 0.94920 & 0.885 & $3 d^{5}\left(a^{2} G\right) 4 p$ & ${ }^{3} G^{o}$ & 0.94050 & 0.874 \\
\hline $3 d^{5}\left(b^{2} F\right) 4 p$ & ${ }^{3} F^{o}$ & 0.93400 & 0.861 & $3 d^{5}\left(b^{2} F\right) 4 p$ & ${ }^{3} G^{o}$ & 0.91153 & 0.846 \\
\hline $3 d^{5}\left(b^{2} F\right) 4 p$ & ${ }^{3} D^{o}$ & 0.90768 & 0.841 & $3 d^{5}\left({ }^{2} S\right) 4 p$ & ${ }^{3} P^{o}$ & 0.89299 & 0.825 \\
\hline $3 d^{5}\left(b^{2} D\right) 4 p$ & ${ }^{3} F^{o}$ & 0.81157 & 0.746 & $3 d^{5}\left(b^{2} D\right) 4 p$ & ${ }^{3} D^{o}$ & 0.80819 & 0.748 \\
\hline $3 d^{5}\left(b^{2} D\right) 4 p$ & ${ }^{3} P^{o}$ & $0.79434 \mathrm{Ek}$ & 0.723 & $3 d^{5}\left(b^{2} G\right) 4 p$ & ${ }^{3} H^{o}$ & 0.74034 & 0.664 \\
\hline $3 d^{5}\left(b^{2} G\right) 4 p$ & ${ }^{3} F^{o}$ & 0.73557 & 0.657 & $3 d^{5}\left(b^{2} G\right) 4 p$ & ${ }^{3} G^{o}$ & 0.72907 & 0.652 \\
\hline $3 d^{5}\left({ }^{2} P\right) 4 p$ & ${ }^{3} P^{o}$ & $0.62122 \mathrm{Ek}$ & 0.544 & $3 d^{5}\left({ }^{4} G\right) 4 d$ & ${ }^{3} F^{e}$ & 0.59060 & 0.520 \\
\hline $3 d^{5}\left({ }^{4} G\right) 4 d$ & ${ }^{3} I^{e}$ & 0.58673 & 0.537 & $3 d^{5}\left({ }^{2} P\right) 4 p$ & ${ }^{3} D^{o}$ & $0.58526 \mathrm{Ek}$ & 0.500 \\
\hline $3 d^{5}\left({ }^{4} G\right) 5 s$ & ${ }^{3} G^{e}$ & 0.58114 & 0.568 & $3 d^{5}\left({ }^{2} P\right) 4 p$ & ${ }^{3} S^{o}$ & $0.56596 \mathrm{Ek}$ & 0.476 \\
\hline $3 d^{5}\left({ }^{4} D\right) 4 d$ & ${ }^{3} G^{e}$ & 0.53051 & 0.526 & $3 d^{5}\left({ }^{4} D\right) 5 s$ & ${ }^{3} D^{e}$ & 0.52381 & 0.507 \\
\hline $3 d^{5}\left(c^{2} D\right) 4 p$ & ${ }^{3} F^{o}$ & $0.50945 \mathrm{Ek}$ & 0.433 & $3 d^{5}\left(c^{2} D\right) 4 p$ & ${ }^{3} D^{o}$ & $0.49426 \mathrm{Ek}$ & 0.420 \\
\hline $3 d^{5}\left({ }^{2} I\right) 5 s$ & ${ }^{3} I^{e}$ & 0.45865 & 0.446 & $3 d^{5}\left({ }^{4} G\right) 5 p$ & ${ }^{3} F^{o}$ & 0.43399 & 0.423 \\
\hline $3 d^{5}\left({ }^{4} G\right) 5 p$ & ${ }^{3} H^{o}$ & 0.43338 & 0.422 & $3 d^{5}\left({ }^{4} G\right) 5 p$ & ${ }^{3} G^{o}$ & 0.42559 & 0.412 \\
\hline $3 d^{5}\left({ }^{4} D\right) 5 p$ & ${ }^{3} D^{o}$ & 0.37360 & 0.362 & $3 d^{5}\left({ }^{4} D\right) 5 p$ & ${ }^{3} F^{o}$ & 0.37290 & 0.358 \\
\hline $3 d^{5}\left({ }^{2} I\right) 5 p$ & ${ }^{3} I^{o}$ & 0.30724 & 0.294 & $3 d^{5}\left({ }^{2} I\right) 5 p$ & ${ }^{3} H^{o}$ & 0.30266 & 0.289 \\
\hline $3 d^{5}\left({ }^{4} G\right) 6 s$ & ${ }^{3} G^{e}$ & 0.21115 & 0.207 & $3 d^{5}\left({ }^{4} D\right) 6 s$ & ${ }^{3} D^{e}$ & 0.15486 & 0.147 \\
\hline $3 d^{6}$ & ${ }^{1} I^{e}$ & 1.97620 & 1.815 & $3 d^{6}$ & ${ }^{1} G^{e} 2$ & 1.97137 & 1.836 \\
\hline $3 d^{6}$ & ${ }^{1} S^{e} 2$ & 1.93560 & 1.820 & $3 d^{6}$ & ${ }^{1} D^{e} 2$ & 1.92656 & 1.793 \\
\hline $3 d^{6}$ & ${ }^{1} F^{e}$ & 1.86192 & 1.717 & $3 d^{6}$ & ${ }^{1} G^{e} 1$ & 1.73139 & 1.581 \\
\hline $3 d^{6}$ & $b^{1} D^{e}$ & $1.55075 \mathrm{Ek}$ & 1.376 & $3 d^{5}\left({ }^{2} I\right) 4 s$ & ${ }^{1} I^{e}$ & 1.49256 & 1.365 \\
\hline $3 d^{5}\left({ }^{2} D_{3}\right) 4 s$ & ${ }^{1} D^{e}$ & 1.46142 & 1.400 & $3 d^{5}\left({ }^{2} F_{2}\right) 4 s$ & ${ }^{1} F^{e}$ & 1.45181 & 1.360 \\
\hline $3 d^{5}\left({ }^{2} H\right) 4 s$ & ${ }^{1} H^{e}$ & 1.40969 & 1.298 & $3 d^{5}\left({ }^{2} G_{2}\right) 4 s$ & ${ }^{1} G^{e}$ & 1.40068 & 1.288 \\
\hline $3 d^{5}\left({ }^{2} F_{1}\right) 4 s$ & ${ }^{1} F^{e}$ & 1.36852 & 1.258 & $3 d^{5}\left({ }^{2} S\right) 4 s$ & ${ }^{1} S^{e}$ & $1.31960 \mathrm{Ek}$ & 1.201 \\
\hline $3 d^{5}\left({ }^{2} D_{2}\right) 4 s$ & ${ }^{1} D^{e}$ & 1.25435 & 1.151 & $3 d^{5}\left(a^{2} D\right) 4 p$ & ${ }^{1} F^{o}$ & 1.24115 & 0.976 \\
\hline $3 d^{5}\left(a^{2} D\right) 4 p$ & ${ }^{1} D^{o}$ & 1.19781 & 1.007 & $3 d^{5}\left({ }^{2} G_{1}\right) 4 s$ & ${ }^{1} G^{e}$ & 1.17799 & 1.053 \\
\hline $3 d^{5}\left({ }^{2} I\right) 4 p$ & ${ }^{1} H^{o}$ & 1.05259 & 0.983 & $3 d^{5}\left({ }^{2} I\right) 4 p$ & ${ }^{1} K^{o}$ & 1.05003 & 0.982 \\
\hline $3 d^{5}\left(a^{2} F\right) 4 p$ & ${ }^{1} G^{o}$ & 1.02845 & 0.979 & $3 d^{5}\left({ }^{2} P\right) 4 s$ & ${ }^{1} P^{e}$ & $1.02769 \mathrm{Ek}$ & 0.907 \\
\hline $3 d^{5}\left({ }^{2} I\right) 4 p$ & ${ }^{1} I^{o}$ & 1.01588 & 0.942 & $3 d^{5}\left(a^{2} D\right) 4 p$ & ${ }^{1} P^{o}$ & 0.98898 & 0.947 \\
\hline $3 d^{5}\left(a^{2} F\right) 4 p$ & ${ }^{1} D^{o}$ & 0.97920 & 0.931 & $3 d^{5}\left(a^{2} G\right) 4 p$ & ${ }^{1} G^{o}$ & 0.97863 & 0.917 \\
\hline $3 d^{5}\left(a^{2} F\right) 4 p$ & ${ }^{1} F^{o}$ & 0.97293 & 0.924 & $3 d^{5}\left({ }^{2} H\right) 4 p$ & ${ }^{1} I^{o}$ & 0.96303 & 0.908 \\
\hline $3 d^{5}\left({ }^{2} D\right) 4 s$ & ${ }^{1} D^{e}$ & $0.95464 \mathrm{Ek}$ & 0.836 & $3 d^{5}\left(a^{2} G\right) 4 p$ & ${ }^{1} H^{o}$ & 0.93526 & 0.874 \\
\hline $3 d^{5}\left({ }^{2} H\right) 4 p$ & ${ }^{1} H^{o}$ & 0.93292 & 0.862 & $3 d^{5}\left(a^{2} G\right) 4 p$ & ${ }^{1} F^{o}$ & 0.93114 & 0.858 \\
\hline $3 d^{5}\left(b^{2} F\right) 4 p$ & ${ }^{1} D^{o}$ & 0.92586 & 0.857 & $3 d^{5}\left(b^{2} F\right) 4 p$ & ${ }^{1} G^{o}$ & 0.89492 & 0.825 \\
\hline $3 d^{5}\left(b^{2} F\right) 4 p$ & ${ }^{1} F^{o}$ & 0.87996 & 0.800 & $3 d^{5}\left({ }^{2} S\right) 4 p$ & ${ }^{1} P^{o}$ & 0.87101 & 0.796 \\
\hline $3 d^{5}\left(b^{2} D\right) 4 p$ & ${ }^{1} F^{o}$ & 0.79942 & 0.735 & $3 d^{5}\left(b^{2} D\right) 4 p$ & ${ }^{1} P^{o}$ & $0.78552 \mathrm{Ek}$ & 0.718 \\
\hline $3 d^{5}\left(b^{2} D\right) 4 p$ & ${ }^{1} D^{o}$ & 0.77580 & 0.710 & $3 d^{5}\left(b^{2} G\right) 4 p$ & ${ }^{1} H^{o}$ & 0.71479 & 0.637 \\
\hline $3 d^{5}\left(b^{2} G\right) 4 p$ & ${ }^{1} G^{o}$ & 0.71026 & 0.630 & $3 d^{5}\left(b^{2} G\right) 4 p$ & ${ }^{1} F^{o}$ & 0.70085 & 0.618 \\
\hline $3 d^{5}\left({ }^{2} P\right) 4 p$ & ${ }^{1} D^{o}$ & 0.57862 & 0.499 & $3 d^{5}\left(c^{2} D\right) 4 p$ & ${ }^{1} D^{o}$ & 0.48902 & 0.404 \\
\hline $3 d^{5}\left(c^{2} D\right) 4 p$ & ${ }^{1} F^{o}$ & 0.47730 & 0.398 & & & & \\
\hline
\end{tabular}

${ }^{\mathrm{a}}$ The term energy is from an incomplete set of observed fine-structure levels.

levels. Identification is carried out through detailed examination of the effective quantum number of the states employing the code ELEVID [10]. Oscillator strengths for transitions among all computed bound states are obtained. The photoionization cross sections are calculated for all bound states below the ionization threshold. The resonances in the cross sections are delineated for the Rydberg series corresponding to each target state of Fe IV, characterized by the effective quantum number $\nu$, up to $\nu=10$ below each target state. The resonances in the small energy region between $\nu>10$ and 
the corresponding target threshold are averaged in the Gailitis averaging procedure [11].

The computations were carried on the Cray Y-MP computer. As for Fe II, it was necessary to divide the calculations into small groups of few symmetries, $S L \pi$, at a time because of the large amount of memory, disk space, and CPU time required. The largest cases were for the singlet and the triplet spin symmetries, $(2 S+1)=1,3$. The largest size of the Hamiltonian matrix was about 2000, with 150 channels, requiring about 1.3 Gbytes of computer disk space for each single continuum wave function and 32 Mbytes of RAM memory. The total CPU time required for the computations was $500 \mathrm{~h}$ with another $400 \mathrm{~h}$ for code development, trials, technical problems, etc.

\section{RESULTS AND DISCUSSIONS}

We present the results for calculated energies, oscillator strengths, and photoionization cross sections of Fe III in the three subsections below.

\section{A. Energy levels}

The first set of calculations is for the bound states of the system $e+$ Fe IV $\rightarrow$ Fe III. We obtain 805 LS bound states of Fe III, about four times more than observed in the laboratory $[12,13]$, which lie below the first ionization threshold $3 d^{5}\left({ }^{6} S\right)$. The total number of $L S$ bound states is 2381 , corresponding to $S_{t} L_{t} \pi_{t} n \iota$, where $S_{t} L_{t} \pi_{t}$ are the 49 target states and $n$ goes from the ground state to 10 (11 for a few symmetries), and $0 \leqslant \iota \leqslant 9$. The states that lie above the first ionization threshold are usually quasibound autoionizing states, but may be bound states in pure $L S$ coupling; we do not consider such states in the present work. All the observed $199 L S$ terms $[12,13]$ are identified, indicating an adequate and reasonably complete representation for the wavefunction expansion $\Psi$. The National Institute of Standards and Technology table [12] contains 184 observed $L S$ terms, while the recent measurements at Lund [13] find 15 additional $L S$ terms. Of the 15 new observed terms, two states, $3 d^{6}\left({ }^{1} D\right)$ and $3 d^{4} 4 s\left({ }^{1} S\right)$, were predicted by our earlier calculations [4]. The calculated energy values are compared with the observed ones in Table II. The table lists the observed terms statistically averaged over the fine-structure levels. The calculated binding energy of the ground state of Fe III is $2.127 \mathrm{Ry}$, compared to the observed value of 2.2528 Ry, a difference of $5.6 \%$. For most of the calculated energies the difference with the observed ones is within 7\%; the largest difference is $21.4 \%$ for the $3 d^{5}\left(a^{2} D\right) 4 p\left({ }^{1} F^{o}\right)$ state, which has a large percentage of admixture with other states. Inclusion of relativistic effects and higher configuration interactions should further improve the calculated energies, but considerably more computational effort would be needed for such $a b$ initio calculations [4].

\section{B. Oscillator strengths}

The oscillator strengths, or the $f$ values, of Fe III are obtained for the bound-bound transitions between all bound $L S$ terms considered in this work. The total number of $f$ values calculated is 249584 , corresponding to all states $S_{t} L_{t} \pi_{t} n \ell$, with $n \leqslant 10$, and $0 \leqslant \ell \leqslant 9$. Of these 11979 cor- respond to the states that lie below the ionization threshold. Currently available $f$ values in the literature are from calculations by Sawey and Berrington [2], Kurucz and Peytremann [14], Fawcett [16], Biemont [15], and Ekberg [13]. Except for the first, which used the CC approximation for the OP work, all the rest of the references used variations of the Cowan's code [17] and agree well with each other. The present work will be compared with two of them through lifetime values. Although no measured $f$ values are available for comparison, the lifetimes of four states of Fe III are available that were measured by Andersen, Petersen, and Biemont [18] The lifetime of a state $j$ is the reciprocal of the sum of the transition probabilities, $A_{j i}$, to all lower states $i$. The transition probability $A_{j i}$ between states $i$ and $j$ is related to the oscillator strength, $f_{i j}$, as $A_{j i}=1 / 2 \alpha^{3}\left(g_{i} / g_{j}\right) E_{j i}^{2} f_{i j}$, where $\alpha$ is the fine-structure constant, $g_{i}$ and $g_{j}$ are statistical weight factors, and $E_{j i}$ is the transition energy. Table III presents the calculated lifetimes of the four states and compares with the measured ones. The present lifetimes are obtained from the calculated $A$ values where the observed energy differences have been used for the transition energy instead of the calculated ones. In Table III, the BiemontKurucz-Peytremann (BKP) values correspond to the calculated lifetimes of Andersen, Petersen, and Biemont [18] where they used the $f$ values for strong spectral lines calculated by Biemont [15], and weak lines calculated by Kurucz and Peytremann [14]. The present lifetimes of states $z^{5} H^{o}$ and $z^{5} G^{o}$ agree within experimental uncertainties of Andersen, Petersen, and Biemont, and for the state $z^{3} H^{o}$ we agree with the BKP value, which is lower than the measured value. The present lifetime of state $y^{1} I^{o}$ is lower than the measured value and the BKP value (the latter two agree with each other). The reason for the present lower value could be because of the relatively high angular momentum of the state ( $L=6$ ) for which it is often difficult to (i) include sufficient correlation from other states, and (ii) employ a sufficiently large $R$-matrix boundary to represent the bound-state wave function in the inner region for the high- $L$ states.

\section{Photoionization cross sections}

\section{Total photoionization cross sections}

Total photoionization cross sections $\sigma_{\mathrm{PI}}$, including detailed autoionizing resonances, for the 805 bound states are obtained. By "total" we mean the sum of all individual cross sections leaving the residual ion in all of the coupled states of Fe IV (out of the 49 target states considered). Some specific results are presented that describe the important features in the photoionization cross sections.

As the energy of the photons increases, crossing various excited thresholds of the target, the photoionization cross

TABLE III. Lifetimes, $\tau$ (ns), of Fe III. The experimental values are from Andersen, Petersen, and Biemont [18].

\begin{tabular}{lccc}
\hline \hline State & Present & $\begin{array}{c}\text { Lifetime } \\
\text { Expt }\end{array}$ & BKP \\
\hline$z^{5} H^{o}$ & 1.9 & $1.6(0.3)$ & 1.7 \\
$z^{5} G^{o}$ & 2.1 & $2.1(0.3)$ & 2.0 \\
$z^{3} H^{o}$ & 1.4 & $1.7(0.2)$ & 1.4 \\
$y^{1} I^{o}$ & 0.82 & $1.2(0.2)$ & 1.2 \\
\hline \hline
\end{tabular}



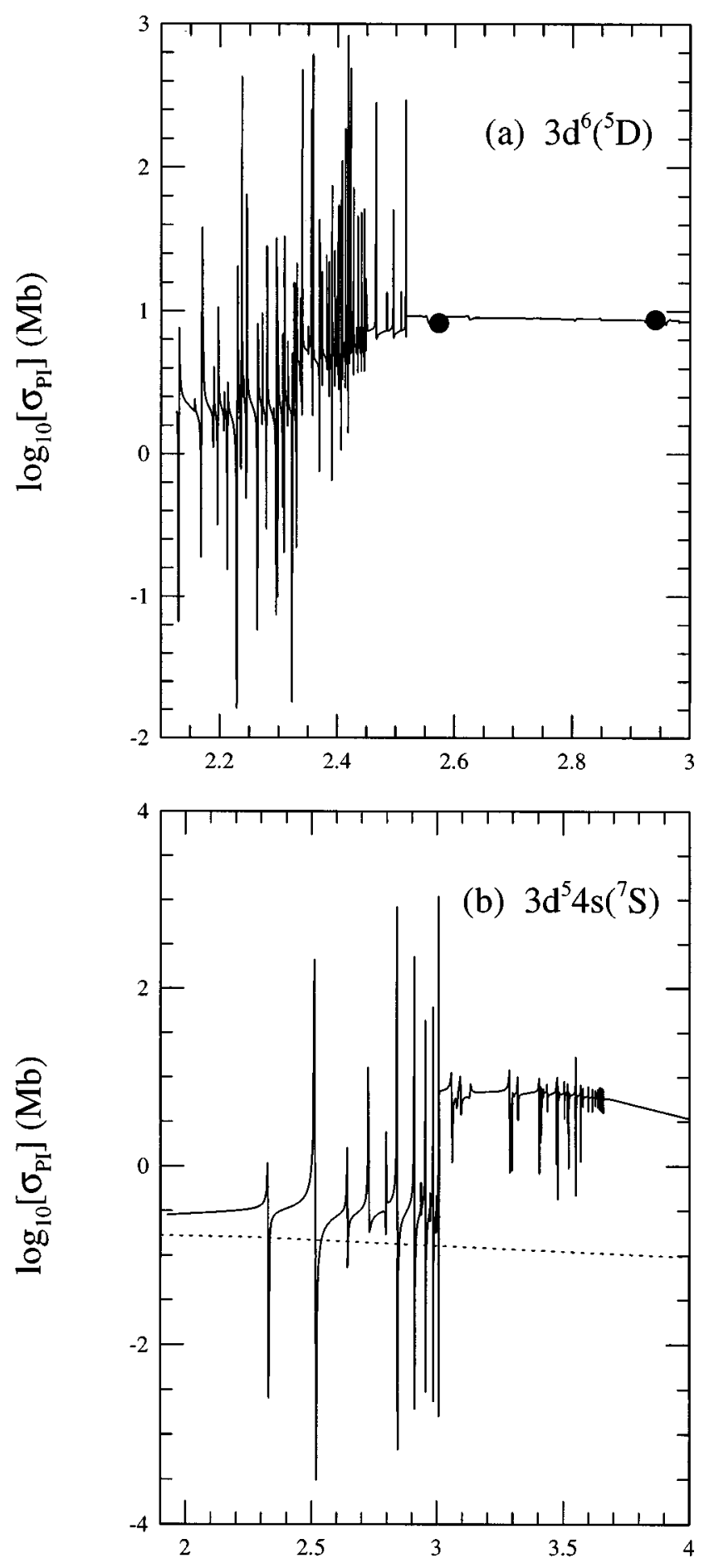

Photon Energy (Ry)

FIG. 1. Photoionization cross section $\sigma_{\text {PI }}$ (solid lines) of (a) the ground state $3 d^{6}\left({ }^{5} D\right)$ and (b) excited state $3 d^{5} 4 s\left({ }^{7} S\right)$ of Fe III. The filled circles in (a) are from Ref. [19] and the dotted curve in (b) is from the 16-state CC calculations of Ref. [2].

sections show resonances due to the autoionizing Rydberg series of states belonging to each target state. The background cross sections due to direct ionization by absorption of photons can be affected considerably by these autoionizing resonances. Figure 1 presents such examples where the

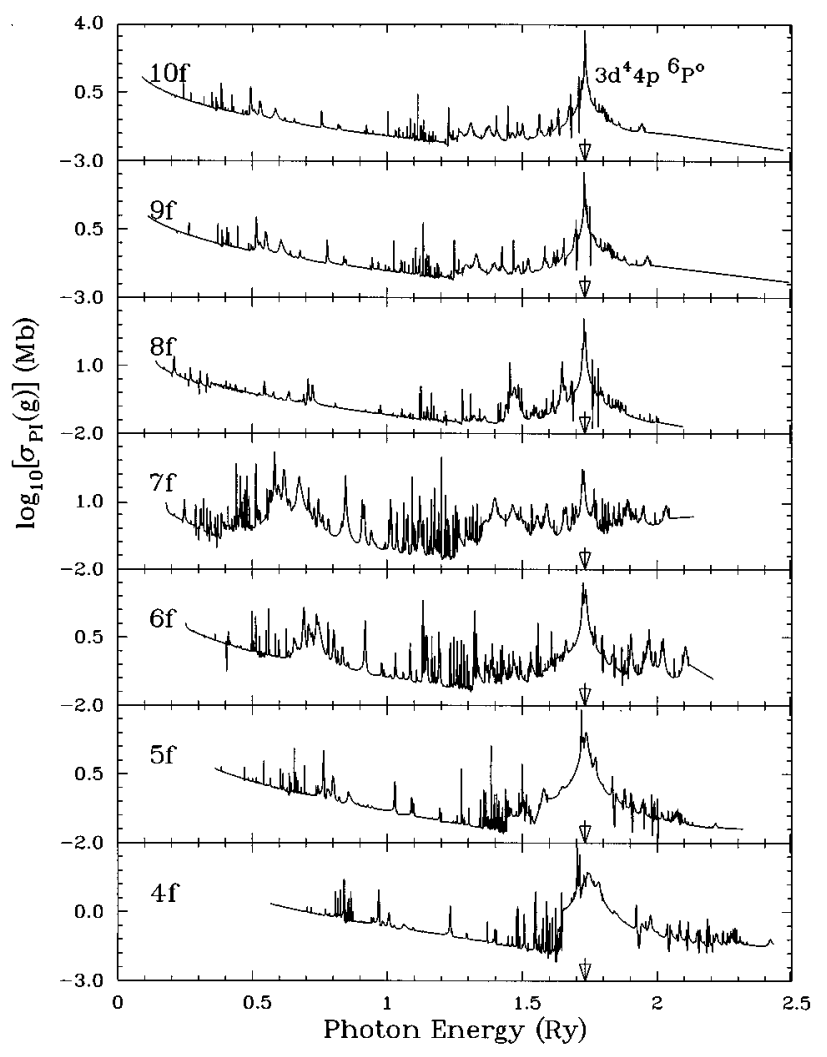

FIG. 2. Photoionization cross sections $\sigma_{\mathrm{PI}}$ of the Rydberg series of bound states of Fe III: $3 d^{56} \operatorname{Snf}\left({ }^{5} F^{o}\right), 4 \leqslant n \leqslant 10$. The PEC resonance, indicated by the arrow in each panel, corresponds to the dipole core transition $3 d^{5}\left({ }^{6} S\right) \rightarrow 3 d^{4}{ }^{5} 4 p\left({ }^{6} P^{o}\right)$ in Fe IV. (With the exception of the top panel, the upper limit is not shown on the $y$ axis.)

photoionization cross sections for (a) the $3 d^{6}\left({ }^{5} D\right)$ ground state, and (b) the $3 d^{5} 4 s\left({ }^{7} S\right)$ state of Fe III are shown. In Fig. 1(a) the present ground-state cross sections show a large number of resonances in the low-energy region, whereas the higher-energy region is comparatively smooth since the coupling effects from the high-lying $3 d^{4} 4 s$ and $3 d^{4} 4 p$ states are weaker. These cross sections show patterns similar to the earlier OP data [2], except for the high-energy background, which falls slightly faster in the 49-state CC calculations (not shown here). The filled circles in the figure correspond to central-field cross sections [19], which, when extrapolated to the first ionization threshold, would give a threshold value 5 times higher than the present one, thus overestimating the cross sections in the near-threshold region considerably. In Fig. 1(b) the present cross sections (solid curve) for the $3 d^{5} 4 s\left({ }^{7} S\right)$ state are compared with the earlier 16-state OP calculations of Sawey and Berrington [2] (dotted curve), and it is seen that their work misses all of the resonances because their CC eigenfunction expansion did not include higher states that couple to $3 d^{5} 4 s\left({ }^{7} S\right)$.

The narrow resonances in the cross sections usually correspond to the Rydberg series of states $S_{t} L_{t} \pi_{t} \nu \ell$ belonging to the target state $S_{t} L_{t} \pi_{t}$, where $\nu$ is the effective quantum number. But, as mentioned earlier, there may be wide resonances in the cross sections due to PEC $[6,11]$. For this type of resonance the target goes through an allowed dipole transition from the ground state to a higher state, while the outer 


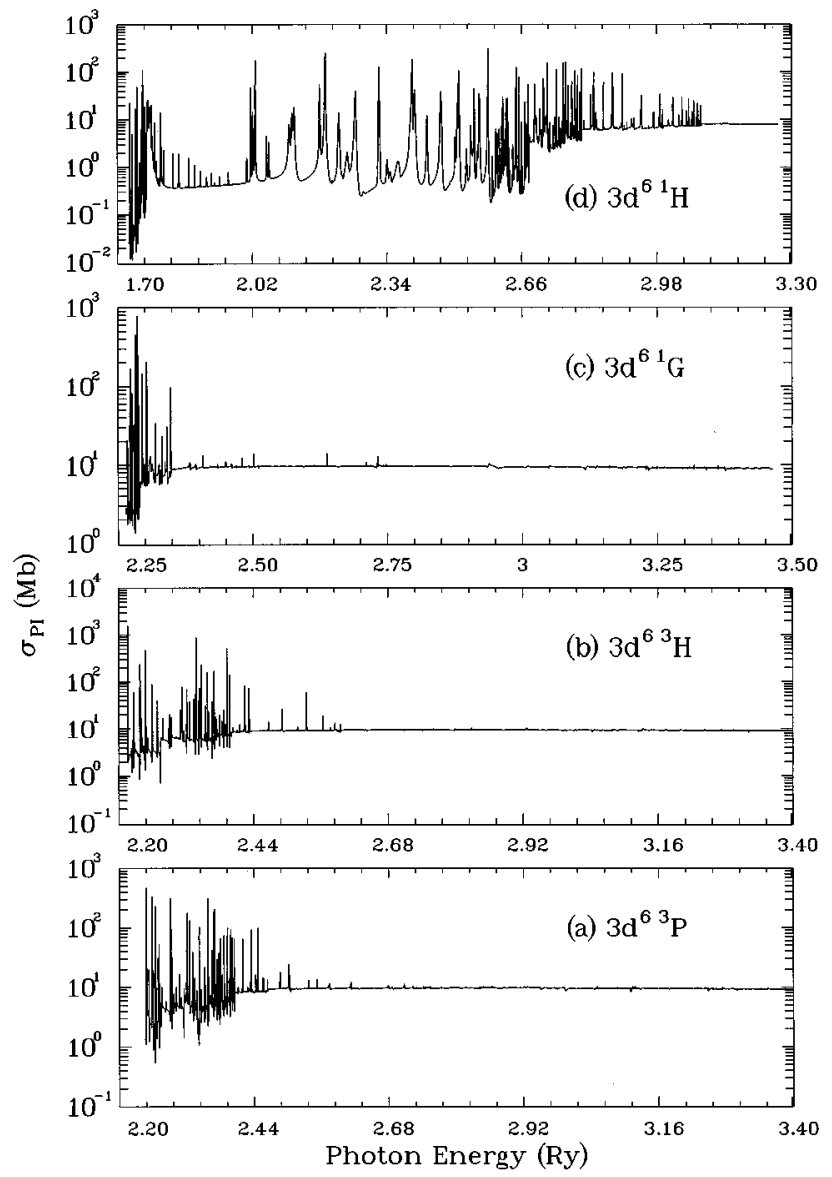

FIG. 3. Photoionization cross sections $\sigma_{\mathrm{PI}}$ for the metastable states (a) $3 d^{6}\left({ }^{3} P\right)$, (b) $3 d^{6}\left({ }^{3} H\right)$, (c) $3 d^{6}\left({ }^{1} G\right)$, and (d) $3 d^{6}\left({ }^{1} H\right)$ of Fe III.

electron remains a "spectator." This effect enhances the cross sections considerably, even by orders of magnitude. PEC resonances are more pronounced in the photoionization cross sections of excited bound states since the outer electron is loosely bound to the ion core. The large PEC resonance due to dipole-allowed core excitation of ground state $3 d^{5}\left({ }^{6} S\right)$ of Fe IV to the excited $3 d^{4} 4 p\left({ }^{6} P^{o}\right)$ state is seen in Fig. 2. The figure shows the photoionization cross sections of seven excited states of Fe III belonging to the Rydberg series $3 d^{56} \operatorname{Snf}\left({ }^{5} F^{o}\right)$, where $n=4-10$. The arrow in each panel of this figure points to the position of the excited ${ }^{6} P^{o}$ state of the core Fe IV, which remains at the same photon energy for each photoionization state of Fe III. This figure also exhibits the very nonhydrogenic behavior of the excited-state cross sections due to the presence of the resonances, especially due to the PEC resonance.

Figure 3 presents $\sigma_{P I}$ for several metastable states of Fe III, (a) $3 d^{6}\left({ }^{3} P\right)$, (b) $3 d^{6}\left({ }^{3} H\right)$, (c) $3 d^{6}\left({ }^{1} G\right)$, and (d) $3 d^{6}\left({ }^{1} H\right)$. All four states show a large number of resonances near the threshold, indicating higher rates of ionization in this region while the high-energy regions show smooth background except for the $3 d^{5}\left({ }^{1} H\right)$ state. Owing to their comparatively long lifetimes, photoionization and recombination from the metastable states are likely to be significant in the calculation of level populations and spectral line intensities from collisional-radiative models.

\section{Partial photoionization cross sections and electron-ion recombination}

This work also reports partial photoionization cross sections, leaving the residul ion in the ground state, of a large number of bound states of Fe III. These are the 239 septet and quintet states of Fe III that can couple to target ground state $3 d^{5}\left({ }^{6} S\right)$. The partial photoionization cross sections are important in the determination of state-specific populations, recombination rates, etc. The present cross sections will be employed for electron-ion recombination of $\mathrm{Fe}$ III in a unified treatment for total electron-ion recombination [7]. The recombination cross sections are related to the photoionization cross sections through detailed balance (the Milne relation). Inclusion of autoionizing resonances in the cross sections thus accounts for both the radiative recombination and the dielectronic recombination (this unified treatment is complemented by calculations for the purely dielectronic recombination from high- $n$ resonances). In the ionization balance equations under plasma equilibrium, one usually employs the total photoionization of the ground state on the one hand, and the inverse process of recombination into all bound states of the ion on the other; thus the partial photoionization cross sections into specific core states of the residual ion are needed.

The partial photoionization cross sections for Fe III, leaving the residual ion Fe IV in various excited states, are obtained for the ${ }^{5} D$ ground state (Fig. 4). The bottom panel in

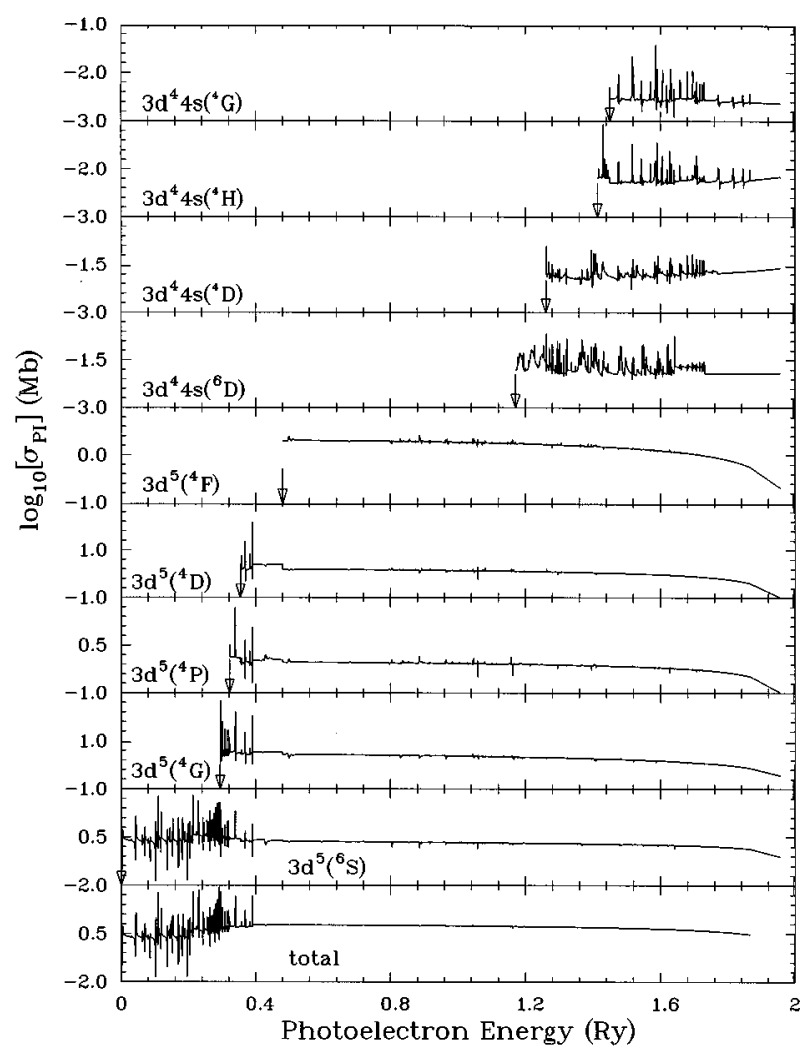

FIG. 4. Partial photoionization cross sections for the ground $3 d^{6}\left({ }^{5} D\right)$ state of Fe III, leaving the Fe IV core in various states, $3 d^{5}\left({ }^{6} S\right), 3 d^{5}\left({ }^{4} G\right), 3 d^{5}\left({ }^{4} P\right), 3 d^{5}\left({ }^{4} D\right)$, and so on, as specified in the panels. The bottommost panel presents the total photoionization cross section. The arrows point to threshold energies of the target states. 
Fig. 4 presents the total photoionization cross section for the state, whereas the upper ones presents the partial photoionization cross sections leaving the target $\mathrm{Fe}$ IV in states $3 d^{5}\left({ }^{6} S\right), 3 d^{5}\left({ }^{4} G\right), 3 d^{5}\left({ }^{4} P\right), 3 d^{5}\left({ }^{4} D\right)$ and so on, as specified in the panels. The arrows point to the threshold energies of these target states. It is evident from the figure that the partial cross section that dominates the total corresponds to that of the first ionization threshold of Fe IV, $3 d^{5}\left({ }^{6} S\right)$. Nonetheless, the resonances in the higher partial cross sections might also contribute significantly to the probability for ionization into those levels.

\section{CONCLUSION}

A comprehensive study of radiative processes in a complex atomic system, such as the ions of the iron group elements, reveals important physical phenomena characteristic of strongly correlated systems. Large-scale computations are reported for the detailed study of radiative processes in Fe III, with computed energy levels, oscillator strengths, and photoionization cross sections, in an ab initio manner. We consider over four times the number of bound states than have been observed in the laboratory. Good agreement is found between the calculated energies and all those that are observed. The accuracy of the oscillator strengths is inferred from the reasonably good agreement with the measured lifetimes of four bound states of Fe III. Total and partial photoionization cross sections (with final states specified) are computed for all the bound states and the resonance structures are delineated in detail. The parameters reported in the present work should be of higher accuracy than the previous OP data for Fe III. It is expected that the new photoionization data in particular will yield more accurate photoionization models than present models, which employ earlier photoionization data. The partial photoionization cross sections will be employed for the calculation of total election-ion recombination rate coefficients of $\mathrm{Fe}$ III.

The complete radiative data of the present work for energy levels, oscillator strengths, and photoinization cross sections of Fe III will be available through the Opacity Project electronic database, TOPbase [20].

\section{ACKNOWLEDGMENTS}

I would like to thank Professor Anil K. Pradhan for contributions (supported by NSF Grant No. PHY-9421898), Manuel Bautista for providing the Fe IV target wave functions, A. F. Robey and Dr. J. R. Fuhr from NIST for providing their most recent compilation of observed energy values of Fe III and Fe IV electronically, and Dr. J. O. Ekberg for his recently measured energy values of some Fe III states. This work was supported by NASA Grants No. NAGW-3315 and No. NAS-32643. The computational work was carried out on the Cray YMP computer at the Ohio Supercomputer Center.
[1] M.J. Seaton, J. Phys. B 20, 6363 (1987).

[2] P.M.J. Sawey and K.A. Berrington, J. Phys. B 25, 1451 (1992).

[3] D.G. Hummer, K.A. Berrington, W. Eissner, A.K. Pradhan, H.E. Saraph, and J.A. Tully, Astron. Astrophys. 279, 298 (1993).

[4] S.N. Nahar and A.K. Pradhan, J. Phys. B 27, 429 (1994).

[5] A.M. Bautista and A.K. Pradhan, J. Phys. B 28, L173 (1995).

[6] Yu Yan and M.J. Seaton, J. Phys. B 20, 6409 (1987).

[7] S.N. Nahar and A.K. Pradhan, Phys. Rev. A 49, 1816 (1994); Astrophys. J. 447, 966 (1995).

[8] W. Eissner, M. Jones, and N. Nussbaumer, Comput. Phys. Commun. 8, 270 (1974).

[9] K.A. Berrington, P.G. Burke, K. Butler, M.J. Seaton, P.J. Storey, K.T. Taylor, and Yu Yan, J. Phys. B 20, 6379 (1987).

[10] S.N. Nahar, Phys. Scr. 293, 967 (1995).

[11] S.N. Nahar and A.K. Pradhan, Phys. Rev. A 44, 2935 (1991).
[12] J. Sugar and C. Corliss, J. Phys. Chem. Ref. Data 14, Suppl. 2 (1985).

[13] J.O. Ekberg, Astron. Astrophys. Suppl. 101, 1 (1993).

[14] R.L. Kurucz and E. Peytremann, Smithsonian Astrophysical Observatory Special Report No. 362, 1975 (unpublished).

[15] E. Biemont, J. Quantum Spectrosc. Radiat. Transfer. 16, 137 (1976).

[16] B.C. Fawcett, At. Data Nucl. Data Tables 41, 181 (1989).

[17] R.D. Cowan, The Theory of Atomic Structure and Spectra (University of California Press, Berkeley, California, 1981).

[18] T. Andersen, P. Petersen, and E. Biemont, J. Quantum. Spectrosc. Radiat. Transfer. 17, 389 (1977).

[19] R.F. Reilman and S.T. Manson, Astrophys. J. Suppl. 40, 815 (1979).

[20] W. Cunto, C. Mendoza, F. Ochsenbein, and C.J. Zeippen, Astron. Astrophys. 275, L5 (1993). 